\title{
A Molecular Determinant of Subtype-Specific Desensitization in Ionotropic Glutamate Receptors
}

\author{
ํㅣㄹ Matthew Alsaloum, ${ }^{3}$ Rashek Kazi, ${ }^{1,2}$ Quan Gan, ${ }^{1}$ Johansen Amin, ${ }^{2}$ and ${ }^{-L o n n i e ~ P . ~ W o l l m u t h ~}{ }^{3,4,5}$ \\ ${ }^{1}$ Graduate Program in Neuroscience, ${ }^{2}$ Medical Scientist Training Program, Departments of ${ }^{3}$ Neurobiology \& Behavior and ${ }^{4}$ Biochemistry \& Cell Biology, \\ and ${ }^{5}$ Center for Nervous System Disorders, Stony Brook University, Stony Brook, New York 11794-5230
}

\begin{abstract}
AMPA and NMDA receptors are glutamate-gated ion channels that mediate fast excitatory synaptic transmission throughout the nervous system. In the continual presence of glutamate, AMPA and NMDA receptors containing the GluN2A or GluN2B subunit enter into a nonconducting, desensitized state that can impact synaptic responses and glutamate-mediated excitotoxicity. The process of desensitization is dramatically different between subtypes, but the basis for these differences is unknown. We generated an extensive sequence alignment of ionotropic glutamate receptors (iGluRs) from diverse animal phyla and identified a highly conserved motif, which we termed the "hydrophobic box," located at the extracellular interface of transmembrane helices. A single position in the hydrophobic box differed between mammalian AMPA and NMDA receptors. Surprisingly, we find that an NMDAR-to-AMPAR exchange mutation at this position in the rat GluN2A or GluN2B subunit had a dramatic and highly specific effect on NMDAR desensitization, making it AMPARlike. In contrast, a reverse exchange mutation in AMPARs had minimal effects on desensitization. These experiments highlight differences in desensitization between iGluR subtypes and the highly specific contribution of the GluN2 subunit to this process.
\end{abstract}

Key words: AMPA receptors; gating; NMDA receptors; sequence alignments; structures

\section{Significance Statement}

Rapid communication between cells in the nervous system depends on ion channels that are directly activated by neurotransmitter molecules. Here, we studied ionotropic glutamate receptors (iGluRs), which are ion channels activated by the neurotransmitter glutamate. By comparing the sequences of a vast number of iGluR proteins from diverse animal species, assisted by available structural information, we identified a highly conserved motif. We showed that a single amino acid difference in this motif between mammalian iGluR subtypes has dramatic effects on receptor function. These results have implications in both the evolution of synaptic function, as well as the role of iGluRs in health and disease.

\section{Introduction}

In the mammalian CNS, fast excitatory synaptic transmission is primarily mediated by ionotropic glutamate receptors (iGluRs), which are ligand-gated ion channels activated by the neurotransmitter glutamate (Traynelis et al., 2010). iGluRs are implicated in

Received July 13, 2015; revised Dec. 28, 2015; accepted Jan. 26, 2016.

Author contributions: M.A., R.K., Q.G., and L.P.W. designed research; M.A., R.K., Q.G., and J.A. performed research; M.A., R.K., Q.G., J.A., and L.P.W. analyzed data; M.A., R.K., Q.G., J.A., and L.P.W. wrote the paper.

This work was supported by a NIH R01 Grant from NINDS (NS088479 to L.P.W.), a NRSA NIH Predoctoral Fellowship from NINDS (NS077541 to R.K.), an American Heart Association Predoctoral Fellowship (Q.G.), and Undergraduate Research and (reative Activities fellowships (M.A.). We thank Dr Maurice Kernan and Kelvin Chan for helpful discussions and/or comments on the paper, and an anonymous reviewer for the suggested cluster and intercluster duration analysis.

The authors declare no competing financial interests.

Correspondence should be addressed to Dr Lonnie P. Wollmuth, Departments of Neurobiology \& Behavior and Biochemistry \& Cell Biology, Center for Nervous System Disorders, Stony Brook University, Stony Brook, NY 117945230. E-mail: Ionnie.wollmuth@stonybrook.edu.

M. Alsaloum's present address: Yale School of Medicine, 333 Cedar Street, New Haven, CT 06520.

DOI:10.1523/JNEUROSCI.2667-15.2016

Copyright $\odot 2016$ the authors $\quad 0270-6474 / 16 / 362617-06 \$ 15.00 / 0$ multiple prevalent diseases, including epilepsy and Parkinson's disease, as well as schizophrenia (Paoletti et al., 2013).

There are two major postsynaptic iGluRs subtypes: AMPA (AMPAR) and NMDA (NMDAR) receptors. AMPARs primarily mediate fast electrical signaling, whereas activation of NMDARs regulates synaptic strength, thereby mediating learning and memory (Hunt and Castillo, 2012). These iGluR subtypes exhibit stark differences in desensitization, a process in which the ion channel enters into a nonconducting state in the presence of agonist. AMPARs display rapid gating kinetics and strong desensitization, whereas NMDARs gate much more slowly and undergo less complete desensitization (Traynelis et al., 2010). AMPAR desensitization impacts the amplitude and time course of the fast component of synaptic currents (Trussell and Fischbach, 1989). On the other hand, NMDAR desensitization may alter the excitotoxicity associated with numerous brain disorders (Choi, 1988; Parsons and Raymond, 2014).

The structural basis underlying these functional differences between subtypes is not well understood. All iGluRs share a com- 
mon general structure. They are tetrameric protein complexes comprised of four membrane-spanning subunits surrounding a central ion permeation pore (Sobolevsky et al., 2009; Karakas and Furukawa, 2014; Lee et al., 2014). A functional AMPAR complex can consist of either identical (homomer) or similar (heteromer) subunits (Traynelis et al., 2010). In contrast, NMDARs are obligate heteromers, requiring two glycinebinding GluN1 subunits and typically two glutamate-binding GluN2 (N2A-N2D) subunits. The structural basis of desensitization in AMPARs involves rearrangements of the dimer interface in the ligand-binding domain (LBD; Sun et al., 2002; Dürr et al., 2014; Meyerson et al., 2014). NMDAR desensitization is not as well understood, and in contrast to AMPARs, appears to be mainly determined by elements in the $\mathrm{N}$-terminal domain (ATD), as well as the transmembrane domain forming the ion channel (Krupp et al., 1998; Villarroel et al., 1998).

We sought to further delineate the molecular basis of desensitization across iGluRs. By aligning the amino acid sequences of iGluR subunits spanning the entire animal kingdom, we identified highly conserved positions, putatively situated at the extracellular interface between transmembrane helices, which we termed the "hydrophobic box" (Fig. 1A). Although highly conserved, the hydrophobic box contains one position that is divergent between mammalian AMPARs and NMDARs. We show that exchanging the AMPAR residue at this position into the GluN2A or GluN2B subunit converts the desensitization phenotype of NMDARs to that comparable to AMPARs. Our findings suggest that the hydrophobic box is a molecular determinant of subtype-specific desensitization in iGluRs.

\section{Materials and Methods}

Multiple sequence alignment. To identify iGluR sequences, we performed a PSI-BLAST search using the homo sapiens GluN1 subunit as an initial query against a nonredundant protein database (Altschul et al., 1997) for sequences originating from animals with length varying between 400 and 1200 aa. The search reached convergence after 11 iterations, returning 1319 sequences, which were screened based on the presence of iGluR-like transmembrane topologies using a TMHMM 2.0 server (Krogh et al., 2001). The result of the topology screening included 1152 sequences encompassing AMPA-, NMDA-, and kainate-subtype like receptors from a wide range of animal phyla, as well as a significant number of unknown or hypothetical gene products. Redundant sequences sharing $100 \%$ identity to each other were pruned, leaving 1047 sequences as the final result.

The Clustal Omega multiple sequence alignment program (Sievers et al., 2011) was used to compare the 1047 iGluR sequences generated by our search. The result of the alignment were manually cleaned for local misalignments and viewed in JalView where each position was given consensus and quality scores.

Mutagenesis and expression. Mutations were made in rat GluN1a (NCBI Protein database accession no. P35439), GluN2A (Q00959), GluN2B (Q00960), GluN2C (Q00961), GluN2D (Q62645), GluA1

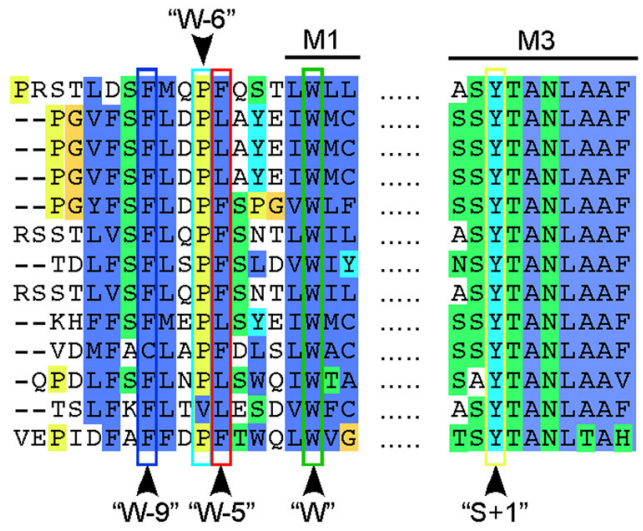

\section{C}

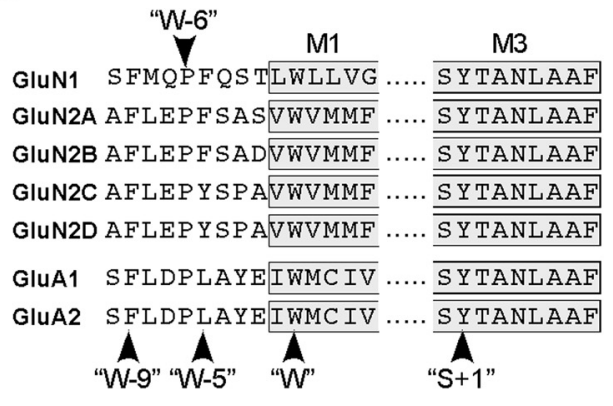

M3

F 48

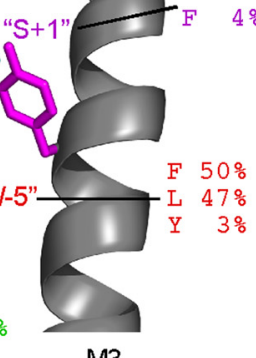$$
\text { (1) }
$$

Figure 1. MSA across iGluR family proteins. $A$, Segments of the MSA of a subset of 1047 animal iGluR subunits around the M (green) in the GluA2 structure (3KG2; Sobolevsky et are indicated. The variable position (W-5) was subject to exchange mutagenesis. We used the GluA2 structure (3KG2) for 列 NMDAR and AMPAR subunits). Position W-5 is an aromatic residue [phenylalanine (F) or tyrosine ( $Y$ )] in NMDAR subunits and $L$ in AMPAR subunits.

(P19490), or GluA2 (P19491) subunits via QuikChange site-directed mutagenesis. cDNA constructs were cotransfected into human embryonic kidney 293 (HEK 293) cells along with a separate pEGFP-Cl vector at a ratio of 4.5:4.5:1 (for NMDARs) or 9:1 (for AMPARs) using $\mathrm{X}$-tremeGene 9 (Roche). To improve survivability, cells were bathed in a media containing the NMDAR competitive antagonist APV $(100 \mu \mathrm{M})$ and $\mathrm{Mg}^{2+}(100 \mu \mathrm{M})$, when transfected with NMDAR subunits, or the competitive antagonist CNQX $(10 \mu \mathrm{M})$, when transfected with AMPAR subunits. All experiments were performed $12-48 \mathrm{~h}$ post-transfection.

Macroscopic current recordings. Macroscopic currents from HEK 293 cells were recorded at room temperature $\left(20^{\circ}-23^{\circ}\right)$ in either the "wholecell" or "outside-out" configuration (Yelshansky et al., 2004). Patch microelectrodes were filled with our standard intracellular solution containing the following (in $\mathrm{mm}$ ): $140 \mathrm{KCl}, 10$ HEPES, 1 BAPTA, pH 7.2 $(\mathrm{KOH})$. The extracellular solution consisted of the following (in $\mathrm{mm}$ ): $140 \mathrm{NaCl}, 1 \mathrm{CaCl}_{2}, 10$ HEPES, pH $7.2(\mathrm{NaOH})$. External solutions were applied using a piezo-driven double barrel application system. For NMDARs, one barrel contained the external solution $+0.1 \mathrm{~mm}$ glycine, whereas the other barrel contained the same solution $+1 \mathrm{~mm}$ glutamate. For AMPARs, we did not include glycine and $6 \mathrm{~mm}$ glutamate was applied instead of $1 \mathrm{~mm}$. Currents were low-pass filtered at $2.9 \mathrm{kHz}$ and sampled at $10 \mathrm{kHz}$. For display, currents were digitally refiltered at 0.5 (NMDAR) or 1 (AMPAR) $\mathrm{kHz}$ and resampled at 1 (NMDAR) or 2 (AMPAR) kHz. Percentage desensitization (\% des) was calculated from the ratio of peak $\left(I_{\text {peak }}\right)$ and steady-state $\left(I_{\mathrm{ss}}\right)$ current amplitudes: $\left(1-\frac{I_{s s}}{I_{\text {peak }}}\right) \times 100 \%$. The rate of desensitization $(\tau)$ was calculated by fitting the decaying phase of currents to either a single (AMPARs) or 
double (NMDARs) exponential function. All currents were measured within 15 min of going whole-cell.

Single-channel recordings. Single-channel currents were recorded in the cell-attached configuration at room temperature using an Axopatch 200B (Molecular Devices), analog filtered at $10 \mathrm{kHz}$ (four-pole Bessel filter), and digitized between 25 and $50 \mathrm{kHz}$. The pipette (external) solution consisted of the following (in $\mathrm{mM}$ ): $150 \mathrm{NaCl}, 2.5 \mathrm{KCl}, 1 \mathrm{EDTA}, 1$ glutamate, 0.1 glycine, 10 HEPES, pH $8.0(\mathrm{NaOH})$.

Analysis of single-channel recordings was comparable to that of Kazi et al. (2014). In all instances, on-cell patches contained a single receptor (Kazi et al., 2015). Clusters were defined by minimizing the false event ratio between the third and fourth closed kinetic state (Kazi et al., 2015).

Statistics. Data analysis was performed using IgorPro, QuB, and Excel. Current properties are presented as mean \pm SEM. We used a two-tailed Student's $t$ test to test for significant differences $(p<0.05)$.

\section{Results \\ Identification of the hydrophobic box, a conserved motif in iGluRs}

Residues highly conserved across species are likely to be involved in critical receptor function (e.g., gating) due to evolutionary selection (Capra and Singh, 2007). To identify functional motifs, we generated a multiple-sequence alignment (MSA) of glutamate receptor subunits from a diverse range of organisms (see Materials and Methods; Fig. 1A). The final alignment consisted of 1047 sequences encompassing all iGluR subtypes.

Of the 4604 positions in the MSA, only 32 showed a consensus score $>80 \%$. The most conserved residue is a tryptophan (W) located at the extracellular end of the M1 segment (W526 in GluA2), which is conserved in $99.7 \%$ of all sequences (Fig. $1 A, B$ ). Conservation of tryptophans may not suggest functional significance per se, because they have a preference for membrane interfaces (Schiffer et al., 1992; Yau et al., 1998). However, in the GluA2 structure (3KG2), W526, referenced as "W", is proximal to two other highly conserved positions: a tyrosine (Y616) in the M3 segment, which is part of the highly conserved SYTANLAAF motif, and more distinctly, a proline (P520) in the S1-M1 linker. In our MSA, this tyrosine, referenced as " $\mathrm{S}+1$ " (Jones et al., 2002), is conserved in $96 \%$ of the sequences. The proline, referenced relative to W526 as "W-6", is conserved in $93 \%$ of the sequences. Further, the $\mathrm{C} \alpha$ of position $\mathrm{W}$ is within $8 \AA$ of three other hydrophobic residues: F517 and L521 in the S1-M1 linker and Y616 $(\mathrm{S}+1)$ in the M3 segment (Fig. 1B). Position F517 of GluA2 ("W-9") is conserved as a phenylalanine in $84.7 \%$ of sequences. L521 ("W-5"), on the other hand, primarily contains either phenylalanine (F) (49.6\%) or leucine (L) $(26.9 \%)$. This variable position will be the focus of exchange mutagenesis (see below). We hypothesized that these four hydrophobic residues constitute a functional motif, which we termed the hydrophobic box.

In mammalian iGluRs, elements of the hydrophobic box are highly conserved across both AMPARs and NMDARs (Fig. 1C), with the exception of one position, W-5. In NMDARs, the residue at position $\mathrm{W}-5$ is an aromatic residue, either a phenylalanine [GluN1 (F540), GluN2A (F534), and GluN2B (F529)] or a tyrosine [GluN2C (Y532) and GluN2D (Y551)]. Interestingly, swapping the residue occupying position $\mathrm{W}-5$ of GluN2A for that of GluN2C, which displays no desensitization, does not result in a concomitant swapping of desensitization phenotypes (Krupp et al., 1998; Villarroel et al., 1998). In contrast, the homologous residue at W-5 in AMPARs is a leucine (L) [GluA1(L517) and GluA2(L521)]. We therefore hypothesized that the $\mathrm{W}-5$ position might contribute to subtype-specific differences in desensitization between AMPARs and NMDARs.

\section{Subtype-exchange mutations in GluN2A or GluN2B but not} GluN1 confer AMPAR-like desensitization to NMDARs

AMPAR and NMDARs exhibit different gating properties (Fig. $2 A$, top, $B$ ). In the absence of auxiliary subunits, AMPARs activate rapidly upon glutamate application and with prolonged glutamate exposure enter into a nonconducting desensitized state (Fig. 2A, top). AMPAR desensitization is nearly complete (GluA1 homomers, \%des $=98.9 \pm 0.3 \%$; mean \pm SEM, $n=$ number of recordings; Fig. $2 F)$ and rapid ( $\tau=3.5 \pm 0.2 \mathrm{~ms}, n=4$; Fig. $2 G)$. NMDARs composed of GluN1/GluN2A exhibit less complete (\%des $=65.9 \pm 0.9 \%$; Fig. $2 B, F$ ) and slower (weighted $\tau=$ $482 \pm 24 \mathrm{~ms}, n=11$; Fig. $2 G$ ) desensitization.

To test the importance of the variable residue occupying position $\mathrm{W}-5$ to receptor function, we performed exchange mutations, replacing the native residue in AMPAR subunits (L) with that in NMDAR subunits (F; GluN1, GluN2A) and vice versa. The exchange mutation in AMPARs, GluA1(L"W-5"L) (Fig. 2A, bottom), as well as GluA2(L"W-5"F), led to a modest but significant decrease in the extent (\%des $=95.3 \pm 0.3 \%)$ and no change in the rate of desensitization (Fig. $2 F, G$ ).

For NMDAR subunits, the exchange mutation in GluN1(F“W-5”L) yielded receptors [GluN1(F540L)/GluN2A] that desensitized to the same extent $(73.9 \pm 2.4 \%)$ as wild-type but more rapidly (weighted $\tau=182 \pm 9 \mathrm{~ms}, n=5$; Fig. $2 F, G$ ). In contrast, the exchange mutation in GluN2A(F“W-5"L) had a dramatic effect on both the extent $(95.6 \pm 0.1 \%)$ and rate (weighted $\tau=73 \pm 2 \mathrm{~ms}, n=16$ ) of desensitization. Indeed, the F-to-L mutation in GluN2A conferred AMPAR-like desensitization onto the receptor in terms of the percentage desensitization (Fig. 2F). However, these receptors still desensitized slower than AMPARs (note the different time calibration in Fig. $2 A-E$ ). A similar effect on desensitization occurred in Glu2B-containing NMDARs but no effect occurred in the GluN2C- or GluN2Dcontaining receptors (Fig. $2 F, G$ ) highlighting desensitization differences between GluN2 subunits (Krupp et al., 1998; Villarroel et al., 1998).

Tetrameric AMPARs have four leucines at the $\mathrm{W}-5$ position, whereas substitutions in individual NMDAR subunits would yield receptors with only two leucines. We therefore tested the double-mutant, GluN1(F“W-5”L)/GluN2A(F“W-5”L; Fig. 2E) to better approximate a tetrameric AMPAR. This receptor also strongly desensitized ( $~ 95 \%$; Fig. $2 \mathrm{~F}$ ) and displayed a rate of desensitization (weighted $\tau=40 \pm 3 \mathrm{~ms}, n=7$ ) significantly faster than that of wild-type GluN1/GluN2A $(\approx 482 \mathrm{~ms})$, as well as GluN1/GluN2A(F“W-5”L) ( $\approx 73 \mathrm{~ms}$; Fig. $2 G)$. Although this construct better reflects AMPARs structurally, the rate of desensitization still remains slower than AMPARs.

\section{Effect of F-to-L exchange mutation on NMDAR gating is subunit-specific}

The results shown in Figure 2 indicate that the impact of the subtypeexchange mutation in NMDARs is subunit-specific. To further clarify this point, we measured the activity of single NMDARs in the on-cell configuration for wild-type and NMDARs containing the F-to-L exchange mutation either in GluN1 or GluN2A (Fig. 3; Table 1). In the on-cell configuration, we include supersaturating concentrations of agonists in the pipette solution such that NMDAR binding sites are generally occupied.

The single-channel profile for wild-type GluN1/GluN2A receptors shows brief periods of activity or clusters separated by seconds-long periods of inactivity (interclusters; Fig. $3 A$ ). Periods of inactivity represent receptor desensitization (Banke and Traynelis, 2003; Popescu and Auerbach, 2003). NMDARs con- 

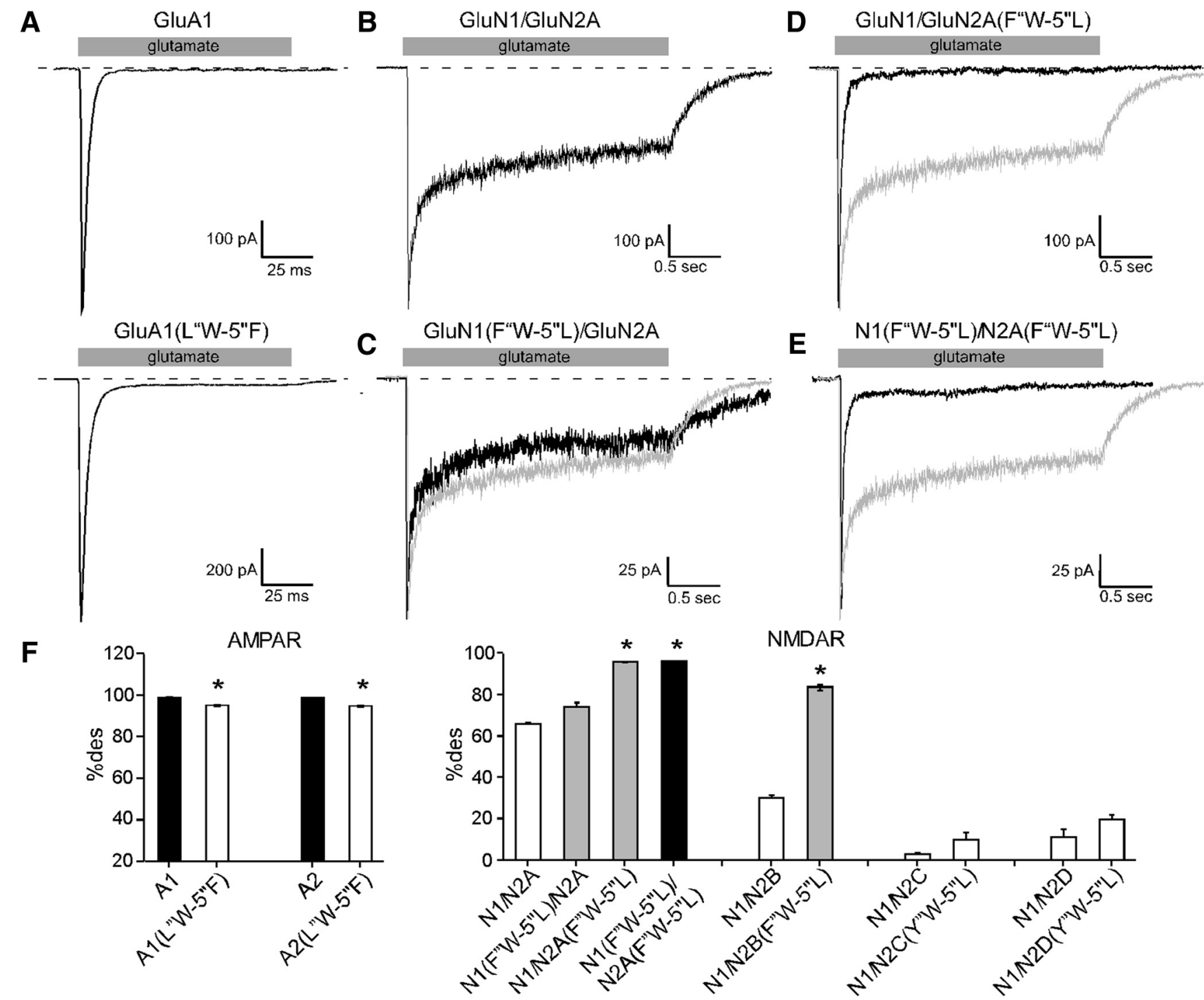

G
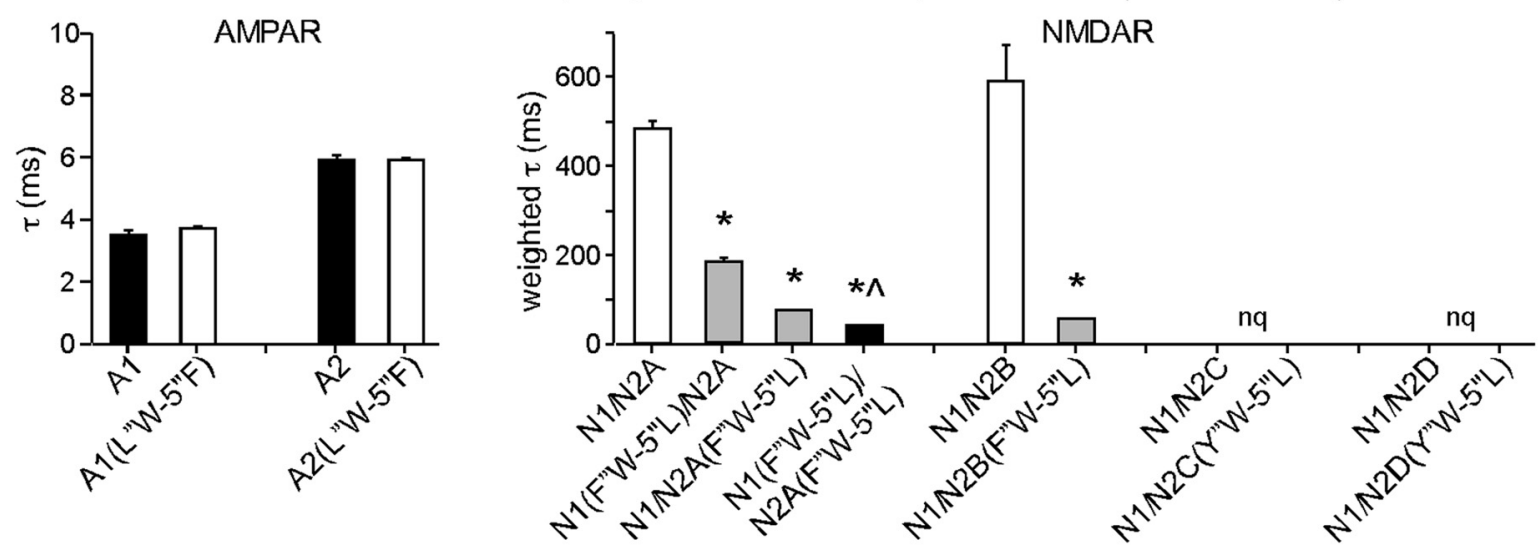

Figure 2. Membrane currents through wild-type and mutant AMPA and NMDA receptors containing exchange mutations at position W-5. A, Outside-out patch recording of membrane currents in HEK 293 cells transfected with wild-type GluA1 (top) or GluA1 containing an exchange mutation at position W-5 (L517F) (bottom). Glutamate (6 mM) was rapidly applied during the shaded box (see Materials and Methods) for $100 \mathrm{~ms}$. Holding potential: $-70 \mathrm{mV}$. B, Whole-cell recording of currents through wild-type GluN1/GluN2A. Glutamate (1 mm; shaded box) was applied for $2.5 \mathrm{~s}$. Cell was continuously bathed in glycine $(0.1 \mathrm{~mm})$. Holding potential: $-70 \mathrm{mV}$. $\boldsymbol{C}-\boldsymbol{E}$, Whole-cell recordings from NMDARs containing exchange mutations at position W-5 for either GluN1 (F540L) (C), GluN2A (F536L) (D), or both subunits (E). Currents recorded as in $\boldsymbol{B}$. Gray current trace is from wild-type GluN1/GluN2A (B). $\boldsymbol{F}, \boldsymbol{G}$, Mean values ( \pm SEM) for percentage desensitization (\%des; $\boldsymbol{F}$ ) and rate of desensitization $(\tau, \mathrm{ms} ; \boldsymbol{G})$. Significance is shown either relative to their respective wild-type construct ${ }^{*}$ ) or for GluN1 (F540L)/GluN2A (F534L), also to (^) GluN1/GluN2A (F534L) ( $p<0.05$, Student's $t$ test). nq, Not quantified. 


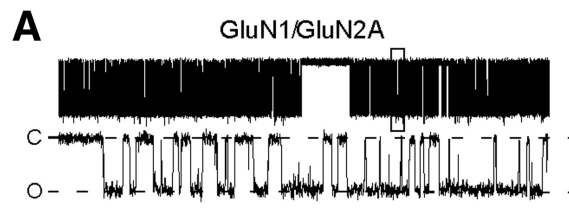

B

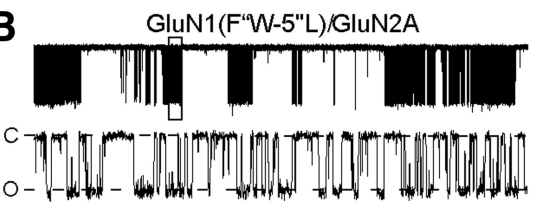

C

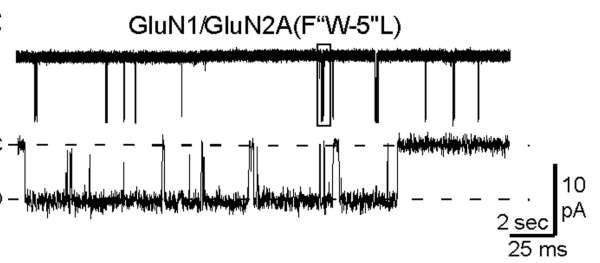

Figure 3. Single-channel recordings of wild-type NMDARs and NMDARs with exchange mutations at position W-5. A-C, Example single-channel recordings of GluN1/GluN2A (A), GluN1 (F540L)/GluN2A (B), and GluN1/GluN2A (F534L) (C). Recordings were performed in the cell-attached configuration with a pipette potential of $+100 \mathrm{mV}$. Downward deflections reflect inward currents. For each trace, the top half shows a low-resolution example (filtered at $1 \mathrm{kHz}$ ) and the bottom half shows a higher resolution portion of the same record (filtered at $3 \mathrm{kHz}$ ). Scale bar for all records is shown in $\boldsymbol{C}$. D, Open $P_{0}$ at equilibrium (solid symbols) or during clusters (open symbols) for GluN1/GluN2A (circles), GluN1 (F540L)/GluN2A (squares), and GluN1/GluN2A (F536L) (triangles). *Indicates significant difference from wild-type. \#Indicates significant difference between equilibrium and cluster $P_{0}(p<0.05)$.

Table 1. Rate constants for entry and recovery from desensitization based on single-channel recordings and macroscopic recovery rates

\begin{tabular}{llllll}
\hline & $\begin{array}{l}\text { Total } \\
\text { events }\end{array}$ & $\begin{array}{l}\text { Entry } \\
\text { rate, } \mathrm{s}^{-1}\end{array}$ & $\begin{array}{l}\text { Recovery } \\
\text { rate, }^{-1}\end{array}$ & $\begin{array}{l}\text { Macroscopic } \\
\text { recovery rate, }\end{array}$ & $\begin{array}{l}\text { 1/recovery } \\
\text { rate, } \mathrm{s}^{-1}\end{array}$ \\
\hline GluN1/GluN2A & $1,995,992$ & $0.07 \pm 0.01$ & $0.48 \pm 0.08$ & $0.92 \pm 0.07$ & $1.08 \pm 0.08$ \\
& $(16)$ & & & $(5)$ & \\
GluN1 (F540L)/ & 812,088 & $0.31 \pm 0.09 *$ & $0.49 \pm 0.08$ & $1.02 \pm 0.06$ & $0.98 \pm 0.06$ \\
GluN2A & $(4)$ & & & $(3)$ & \\
GluN1/ & 25,475 & $2.68 \pm 0.81^{*}$ & $0.13 \pm 0.04^{*} *$ & $4.02 \pm 0.22^{*}$ & $0.25 \pm 0.01^{*}$ \\
GluN2A (F534L) & $(3)$ & & & $(8)$ & \\
\hline
\end{tabular}

Mean values ( \pm SEM) for the entry and recovery rates derived from the reciprocal of the mean cluster and intercluster durations, respectively, from single-channel recordings (Fig. 3). The number of patches is in parentheses below the total number of events. Macroscopic recovery rates from desensitization and their reciprocal (mean $\pm S E M$ ) are shown in the right two columns. The number of recordings is shown in parentheses. Significance is shown relative to GluN1/GluN2A ( ${ }^{*} p<0.05$, Student's $t$ test).

taining the GluN1 F-to-L (Fig. 3B) show decreased activity during clusters (bottom). On the other hand, the GluN2A F-to-L (Fig. 3C) causes extremely long periods of intercluster inactivity with high levels of intracluster activity (bottom).

We quantified equilibrium open probability (equilibrium $P_{\mathrm{o}}$ ), which is a measure of $P_{\mathrm{o}}$ during the entire recording including periods of inactivity, as well as the $P_{\mathrm{o}}$ during clusters (cluster $P_{\mathrm{o}}$; see Materials and Methods). We anticipate that enhanced desensitization will be reflected by decreased equilibrium $P_{\mathrm{o}}$ with minimal or no change in cluster $P_{\mathrm{o}}$. For wild-type GluN1/GluN2A, equilibrium $P_{\mathrm{o}}$ was $\sim 0.7(0.69 \pm 0.03, n=16)$ with, as expected, a higher cluster $P_{\mathrm{o}}(0.81 \pm 0.02$; Fig. $3 D$, circles $)$. The GluN1 F-to-L lowered both the equilibrium $(0.20 \pm 0.03, n=4)$ and cluster $(0.36 \pm 0.01) P_{\mathrm{o}}$ to a similar degree (Fig. $3 D$, squares) indicating that the GluN1 F-to-L strongly dampens NMDAR pore opening. In contrast, the GluN2A F-to-L, relative to wildtype, dramatically reduced equilibrium $P_{\mathrm{o}}$ to $\sim 0.05(0.05 \pm 0.03$, $n=3)$, but had no significant effect on cluster $P_{\mathrm{o}}(0.80 \pm 0.06$; Fig. 3D, triangles) indicating that this mutation in GluN2A selectively affects receptor desensitization while leaving receptor activation intact.
To further define the impact of the F-to-L mutations to desensitization, we characterized cluster and intercluster durations. Because patches contained a single channel, clusters begin when the receptor escapes from the desensitized states and ends when it desensitizes again. Therefore, the reciprocal of the mean cluster and intercluster durations reflect the rate constants for entry into desensitization and recovery from desensitization, respectively (Table 1).

For wild-type (GluN1/GluN2A), based on mean cluster and intercluster durations, the rate of entry into desensitization was $0.07 \pm 0.01 \mathrm{~s}^{-1}$ and the recovery rate was $0.48 \pm 0.08 \mathrm{~s}^{-1}$ (Table 1 ). This entry rate of desensitization is quite slow and most likely reflects that the external solution used in the on-cell recordings, which was distinct from that in the whole-cell recordings (see Materials and Methods), is optimized for channel opening or activation. Indeed, the entry rates, based on mean cluster duration, for GluN1(F“W-5”L) was $0.31 \pm 0.10 \mathrm{~s}^{-1}$ and for GluN2A(F“W-5”L) was $2.68 \pm 0.81 \mathrm{~s}^{-1}$, both significantly faster than that observed for wild-type and paralleling that observed at the whole-cell level (Fig. 2G). Similarly, the recovery rates for GluN1(F“W-5"L) $\left(0.49 \pm 0.08 \mathrm{~s}^{-1}\right)$ was not significantly different from that for wild-type, whereas for GluN2A(F“W-5”L) $\left(0.13 \pm 0.04 \mathrm{~s}^{-1}\right)$ it was significantly slower, again paralleling what was observed at the macroscopic level (Table 1). Thus, for GluN2A(F“W-5”L), the extensive increase in the extent of desensitization (Fig. $2 D, F$ ) is mainly because of the dramatic increased entry rate into desensitization and, to a lesser extent, a slowing of the recovery rate.

\section{Discussion}

In this study, we identified a conserved motif in iGluRs, the hydrophobic box, which contributes subtype-specific gating properties to iGluRs. We found that this motif, and specifically the residue occupying position $\mathrm{W}-5$, can influence the rate and extent of desensitization in NMDARs containing the GluN2A or GluN2B but not GluN2C or GluN2D subunits (Figs. 2, 3; Table 1). In GluN1, the F-to-L mutation did not have a desensitizationspecific effect, as evidenced by a decrease in both equilibrium and cluster $P_{\mathrm{o}}$ (Fig. 3). In contrast, only equilibrium $P_{\mathrm{o}}$ was lowered by the mutation in GluN2A, indicating increased desensitization but intact activation (Fig. 3). In terms of desensitization, the major effect of GluN2A F-to-L was a dramatic increase entry rate (Table 1). Thus, the hydrophobic boxes of GluN1 and GluN2 play distinct roles in NMDAR gating.

Our results are consistent with the idea that the mechanism of desensitization is fundamentally different between AMPARs and NMDARs. The structural and mechanistic basis of AMPAR desensitization involves a rearrangement of the LBD dimer interface (Sun et al., 2002; Meyerson et al., 2014), which seems to play only a minor role in NMDAR desensitization (Borschel et al., 2011). Consistent with the AMPAR desensitization model, we find that when the subtype-exchange mutation, which is independent of the LBD, is introduced into AMPARs, the effect on desensitization was not nearly as dramatic as that in NMDARs composed of GluN1/GluN2A or GluN1/GluN2B (Fig. 2). Hence, 
transmembrane interactions involving the pre-M1 region, as well as M3 and M4 (Ren et al., 2012) appear critical to the mechanism of NMDAR but not that of AMPAR desensitization.

In conclusion, we show that there is a conserved motif that plays a unique role in iGluR desensitization. Our results also suggest distinct mechanisms of desensitization between AMPARs and NMDARs. Although there is significant structural homology, minute differences within the hydrophobic box contributes to the unique desensitization properties between these iGluR subtypes. However, for a model of NMDAR desensitization to be proposed, additional structural elements within the ATD (Krupp et al., 1998; Villarroel et al., 1998), as well as other transmembrane interactions (Ren et al., 2012) need to be investigated. Finally, our experiments have identified a potential tool for studying the impact of NMDAR desensitization on excitotoxicity and synaptic dynamics. Understanding these mechanisms paves the way for future therapeutics in the combat of diseases secondary to iGluR dysfunction.

\section{References}

Altschul SF, Madden TL, Schäffer AA, Zhang J, Zhang Z, Miller W, Lipman DJ (1997) Gapped BLAST and PSI-BLAST: a new generation of protein database search programs. Nucleic Acids Res 25:3389-3402. CrossRef Medline

Banke TG, Traynelis SF (2003) Activation of NR1/NR2B NMDA receptors. Nat Neurosci 6:144-152. CrossRef Medline

Borschel WF, Murthy SE, Kasperek EM, Popescu GK (2011) NMDA receptor activation requires remodelling of intersubunit contacts within ligand-binding heterodimers. Nat Commun 2:498. CrossRef Medline

Capra JA, Singh M (2007) Predicting functionally important residues from sequence conservation. Bioinformatics 23:1875-1882. CrossRef Medline

Choi DW (1988) Glutamate neurotoxicity and diseases of the nervous system. Neuron 1:623-634. CrossRef Medline

Dürr KL, Chen L, Stein RA, De Zorzi R, Folea IM, Walz T, Mchaourab HS, Gouaux E (2014) Structure and dynamics of AMPA receptor GluA2 in resting, pre-open, and desensitized states. Cell 158:778-792. CrossRef Medline

Hunt DL, Castillo PE (2012) Synaptic plasticity of NMDA receptors: mechanisms and functional implications. Curr Opin Neurobiol 22:496-508. CrossRef Medline

Jones KS, VanDongen HM, VanDongen AM (2002) The NMDA receptor M3 segment is a conserved transduction element coupling ligand binding to channel opening. J Neurosci 22:2044-2053. Medline

Karakas E, Furukawa H (2014) Crystal structure of a heterotetrameric NMDA receptor ion channel. Science 344:992-997. CrossRef Medline

Kazi R, Dai J, Sweeney C, Zhou HX, Wollmuth LP (2014) Mechanical coupling maintains the fidelity of NMDA receptor-mediated currents. Nat Neurosci 17:914-922. CrossRef Medline

Kazi R, Daniels M, Wollmuth LP (2015) Assaying the energetics of NMDA receptor pore opening. In: Ionotropic glutamate receptor technologies (Popescu GK, ed), pp 145-162. New York: Humana.
Krogh A, Larsson B, von Heijne G, Sonnhammer EL (2001) Predicting transmembrane protein topology with a hidden Markov model: application to complete genomes. J Mol Biol 305:567-580. CrossRef Medline

Krupp JJ, Vissel B, Heinemann SF, Westbrook GL (1998) N-terminal domains in the NR2 subunit control desensitization of NMDA receptors. Neuron 20:317-327. CrossRef Medline

Lee CH, Lü W, Michel JC, Goehring A, Du J, Song X, Gouaux E (2014) NMDA receptor structures reveal subunit arrangement and pore architecture. Nature 511:191-197. CrossRef Medline

Meyerson JR, Kumar J, Chittori S, Rao P, Pierson J, Bartesaghi A, Mayer ML, Subramaniam S (2014) Structural mechanism of glutamate receptor activation and desensitization. Nature 514:328-334. CrossRef Medline

Paoletti P, Bellone C, Zhou Q (2013) NMDA receptor subunit diversity: impact on receptor properties, synaptic plasticity and disease. Nat Rev Neurosci 14:383-400. CrossRef Medline

Parsons MP, Raymond LA (2014) Extrasynaptic NMDA receptor involvement in central nervous system disorders. Neuron 82:279-293. CrossRef Medline

Popescu G, Auerbach A (2003) Modal gating of NMDA receptors and the shape of their synaptic response. Nat Neurosci 6:476-483. CrossRef Medline

Ren H, Zhao Y, Dwyer DS, Peoples RW (2012) Interactions among positions in the third and fourth membrane-associated domains at the intersubunit interface of the $N$-methyl-D-aspartate receptor forming sites of alcohol action. J Biol Chem 287:27302-27312. CrossRef Medline

Schiffer M, Chang CH, Stevens FJ (1992) The functions of tryptophan residues in membrane proteins. Protein engineering 5:213-214. CrossRef Medline

Sievers F, Wilm A, Dineen D, Gibson TJ, Karplus K, Li W, Lopez R, McWilliam H, Remmert M, Söding J, Thompson JD, Higgins DG (2011) Fast, scalable generation of high-quality protein multiple sequence alignments using Clustal Omega. Mol Syst Biol 7:539. CrossRef Medline

Sobolevsky AI, Rosconi MP, Gouaux E (2009) X-ray structure, symmetry and mechanism of an AMPA-subtype glutamate receptor. Nature 462: 745-756. CrossRef Medline

Sun Y, Olson R, Horning M, Armstrong N, Mayer M, Gouaux E (2002) Mechanism of glutamate receptor desensitization. Nature 417:245-253. CrossRef Medline

Traynelis SF, Wollmuth LP, McBain CJ, Menniti FS, Vance KM, Ogden KK, Hansen KB, Yuan H, Myers SJ, Dingledine R (2010) Glutamate receptor ion channels: structure, regulation, and function. Pharmacol Rev 62: 405-496. CrossRef Medline

Trussell LO, Fischbach GD (1989) Glutamate receptor desensitization and its role in synaptic transmission. Neuron 3:209-218. CrossRef Medline

Villarroel A, Regalado MP, Lerma J (1998) Glycine-independent NMDA receptor desensitization: localization of structural determinants. Neuron 20:329-339. CrossRef Medline

Yau WM, Wimley WC, Gawrisch K, White SH (1998) The preference of tryptophan for membrane interfaces. Biochemistry 37:14713-14718. CrossRef Medline

Yelshansky MV, Sobolevsky AI, Jatzke C, Wollmuth LP (2004) Block of AMPA receptor desensitization by a point mutation outside the ligandbinding domain. J Neurosci 24:4728-4736. CrossRef Medline 